\title{
LAS ARTES DEL ARTE: LAS ARTES LIBERALES \\ EN LA EVOLUCIÓN DEL ARTE LULIANO
}

José Higuera Rubio

IBFCRL - São Paulo

jhiguera@alumni.unav.es

El sepulcro de Ramon Llull, en la iglesia de San Francisco en Palma de Mallorca, descansa sobre unos relieves que representan a las Artes liberales, el trivium y el quadrivium, tal como los describe Jaime Costurer: "con figuras, y follajes, salen de la pared siete bestiones de piedra, de palmo y cuarto de alto, que en sus manos tienen las insignias de la práctica de las artes liberales, y están destinados para sustentar siete estatuas que han de ocupar los nichos que les corresponden (...) de cada uno de ellos salen dos ángeles, que sustentan una corona, destinada para la cabeza de las estatuas (...) en cada una de estas coronas está gravado con letras Góticas antiquísimas el nombre de una de las siete artes liberales con este orden empezando por la parte del altar Gramatica, Logica, Rethorica, Arithmetica, Musica, Geometria, Astrologia" (Costurer, I700, pp. IO-II; ver Fig. I). Ramon Llull se sirvió del modelo de las artes para elaborar su propio Arte, lo que observó Joan Llobet el maestro barcelonés que diseñó el sepulcro a mediados del siglo Xv. Este hecho nos invita a investigar los detalles del proceso que llevó a la inspiración luliana del Arte por las artes.

Desde la antigüedad los términos techne-ars han tenido diversos significados, ya que se les vincula con la adquisición y la aplicación práctica del conocimiento. Asimismo, la habilidad para unir estas facultades está relacionada con la sabiduría (sapientia), la ciencia (scientiae), la filosofía (philosophia) o la prudencia (prudentia). Esta multiplicidad de sentidos se transmite a la Edad Media, de modo que estos términos se convierten en sinónimos y, a veces, se definen mutuamente. Esto sucedía en los diálogos platónicos en los que techne ocupaba el lugar de episteme y al contrario, un uso que Aristóteles criticó e intentó diferenciar. En la República (VII, 522d; 527b) techne es una aplicación práctica que obedece a un proceso racional, así como en la geometría se calculan las proporciones de un conjunto de magnitudes. Aristóteles afirmó en la Ética a Nicómaco (VI, II4Oa) que no hay una operación práctica sin un contenido racional, aunque trató de marcar la diferencia entre el conocimiento especulativo y las operaciones que aplican principios previamente conocidos. El logicismo de los estoicos les llevó a 
definir techne como "el conjunto de representaciones comprehensivas aplicado a la búsqueda de un fin útil para la vida”. Cicerón (Diomedes, I857, p. 24I) elaboró una versión latina de esta definición en la que aparece ars como traducción de techne. En esta definición las "representaciones comprehensivas" se denominan "preceptos", un término que en el siglo XIII adquiere el sentido de principio. La definición ciceroniana de ars como "el orden de principios (praeceptionum) aplicables a un fin útil” es transmitida por la tradición medieval junto a la cuestión acerca de la distinción y/o jerarquía que tienen los principios en la clasificación de las artes liberales y mecánicas, y en relación a las ciencias como la teología, el derecho o la medicina.

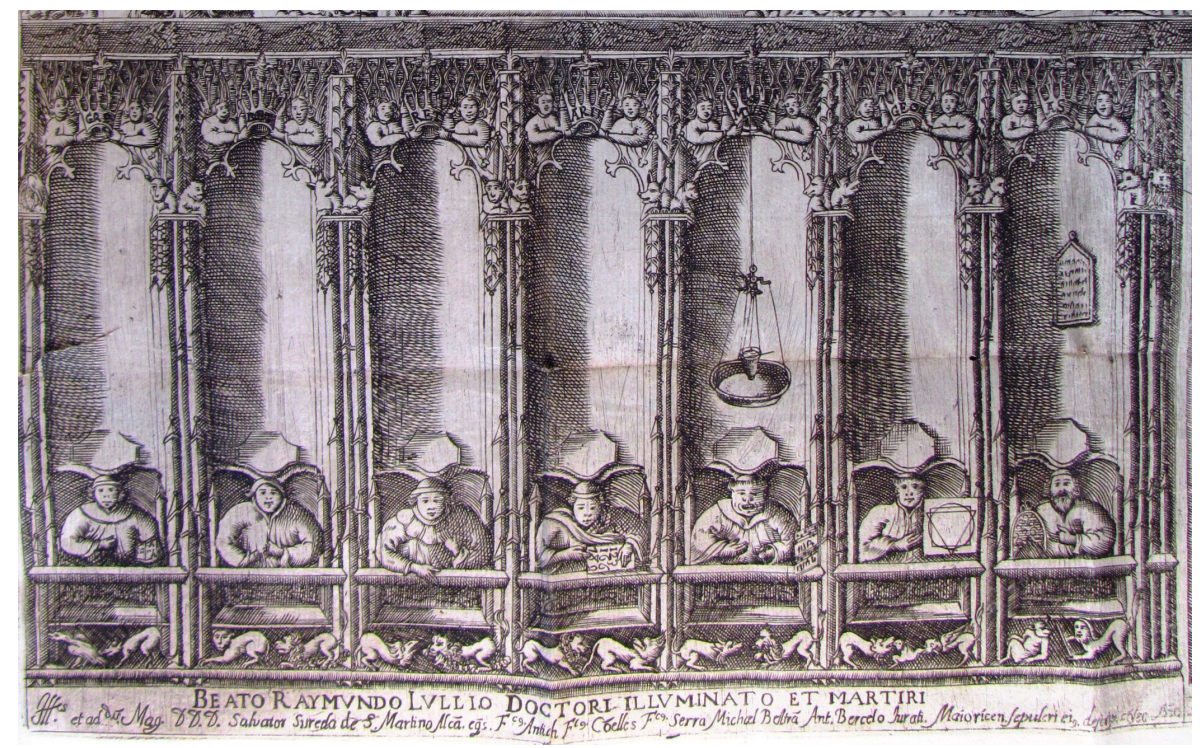

Fig. I. Detalle del grabado del sepulcro de Ramon Llull en la Iglesia San Francisco, en Palma de Mallorca, en el que aparecen las sietes artes liberales (Costurer, I700, I4-I5): Gramatica, Lagica, Rethorica, Arithmetica, Musica, Geometria, Astrologia (Fotografía tomada del volumen que pertenece al Fondo Antiguo de la Biblioteca de la Universidad de Navarra).

La división de las ciencias aparece en la distinción platónica entre la ética, el estudio de la naturaleza y la lógica, así como en la división aristotélica entre ciencias teóricas y prácticas. En la primera están la física, la matemática y la metafísica; y en la segunda la ética, la economía y la política. Boecio se encargaría en el De trinitate de impulsar el énfasis medieval en las ciencias que más se ajustan al estudio de lo divino, por medio de la distinción de los objetos de cada disciplina: 
las ciencias especulativas estudian los objetos separados de la materia que carecen de movimiento, mientras la física estudia los objetos unidos a la materia y al movimiento (Boecio, 2000, pp. I68-170).

Estas distinciones encontrarían un reflejo en el conjunto de las Artes liberales descritas por Isidoro de Sevilla, en los textos didascálicos del siglo XII, y en los tratados árabes que estudian la división aristotélica de las ciencias. Con estos recursos los principios de cada disciplina adquieren un sentido operativo desde la perspectiva de las artes liberales, lo que implica el conocimiento de la naturaleza divina y mundana, tanto en el lenguaje natural como en el matemático.

La relación entre ciencias y artes tuvo un reflejo práctico en la interpretación medieval de las formas de gobierno, en el comportamiento moral y en los oficios de las ciudades (mercaderes, agricultores, navegantes, herreros, tejedores, médicos). La sociedad medieval de los siglos XII y XIII que vivió la explosión de las profesiones urbanas, la consolidación de la educación universitaria, así como la diversificación de los roles socio-políticos de la nobleza y el clero, se encuentra con un modelo de formación en el que las artes "liberan" al intelecto humano, y también facilitan la vida práctica. Estas últimas son las artes mechanicas que reúnen los oficios profesionales emergentes en las ciudades. Aunque se trata de artes manuales que están "atadas al cuerpo" son vistas por Buenaventura como un reflejo más de la luz divina que inunda a la naturaleza, porque ve en ellas una imitación humana artificial de las operaciones del creador (Buenaventura, 1996, p. 52).

En la enseñanza universitaria las Artes son la $u i a$, el instrumento para acceder a otras ciencias como la teología, el derecho, o la medicina. En palabras de Girald de Cambrai las artes representan el suelo y las paredes de un edificio sobre el que se erige el techo que representa la teología, otro autor anónimo dice: artes sine divinitate cassae sunt. En este universo cultural de las artes liberales y mecánicas irrumpe la obra filosófica luliana, que elaboró una síntesis de las artes en un Ars major que compila la multiplicidad de principios de las disciplinas medievales, así como su aplicación práctica: el dialogo teológico sapiencial. El Arte luliano es un gran compendio de la sabiduría artística greco-latina que encontró una cristalización en el pensamiento medieval, a la vez filosófico y teológico, pero también científico y tecnológico.

Con suma curiosidad nos encontramos que la definición más conocida de ars dice que es una práctica, una técnica "sin doctrina" (Bonner, 2007, p. 3). Por las definiciones que se conocen de ars extractadas de los textos que circulaban en el studium medieval —Guías del estudiante, Introducciones a la filosofía, o florilegios- se nota que junto a la finalidad práctica del arte aparece el conocimiento de unos principios o una cierta actividad especulativa que ausculta un cierto objeto de estudio. Si comparamos la definición luliana de arte con otras, conocidas en 
la época, se nota que junto a los términos que expresan finalidad ( $f$-finem) están los que significan conocimiento (conaxensa) o principio, lo que resulta útil para comprender la transmisión de la definición clásica de ars en el Arte luliano:

Ars est ordinatio et constitutio cosgnoscendi finem, cuius homo uult habere cognitionem. Doctrina Pueril (Llull, 2009, p. 36I).

Ars est collectio multorum praeceptorum ad unum finem tendentium (Juan de Dacia, I955, p. 9).

Para comprender mejor el Arte luliano convendría tomar como referencia la idea de que ars es ante todo una collectio principiorum vinculada a una finalidad, lo que resulta básico en la definición clásica de techne-ars y que Llull recoge como "orden del conocimiento". Thomas Le Myesier recoge una definición de ars en una lista de definiciones que está en el manuscrito del Electorium (BN París Ms. Lat. 15450) y que parece sintetizar las definiciones citadas: "ars est collectio philosophum priorum et communis recta et rationalis deductio vel ordinatio ad finem inveniendam" (Hillgarth, 1975, p. 350). Estas definiciones son compatibles con el relato de la Uita coetanea en el que se describe cómo, en medio de un ejercicio contemplativo, Llull recibe la súbita inspiración divina del Ars major, cuyo principal componente es una collectio de principios "generales y específicos". Dicha collectio resulta compatible con la definición clásica de techne-ars en la que los principios (praeceptum) están dispuestos según una cierta finalidad que en el relato de la Uita es la de escribir los libros que exponen el arte.

Estos elementos están ausentes de la definición de ars que Jacques Le Goff ofrece en su conocida introducción a la vida académica medieval (Le Goff, I956, p. 60). Dicha definición transmitió una ambigua sinonimia entre ars y técnica, lo que ocultó el componente epistémico. La referencia al "conjunto (collectio) de principios dispuesto hacia un fin" que no aparece en la definición aristotélica que Le Goff cita unas líneas después. Sin embargo, en los textos que circulaban por el studium medieval éste era un componente fundamental del ars. La definición citada antes dejó de lado los múltiples sentidos de la definición de las artes en relación a las ciencias y al pensamiento filosófico, lo que sin duda aisló la recepción que el mismo Llull hace del término ars y las connotaciones que tuvo en su época.

La multiplicidad de sentidos puede constatarse en el vocabulario greco-latino presente en los textos didascálicos del siglo XII, en las introducciones a la filosofía, así como en las anónimas Guías del estudiante y los florilegios medievales. Estos recursos ayudan a sustituir una definición incompleta de ars que ignora la pluralidad de sentidos que tuvo dicho vocablo. De un parte se trata de una via hacia una disciplina de orden superior, la teología, y de otra es una collectio principiorum orientada hacia una finalidad. Por tanto las artes recogen una división de las cien- 
cias que parte de la teología, así como las respectivas collectio de principios que pertenecen a las disciplinas sermocinales, prácticas y especulativas. Esto explica la sinonimia de ars con términos como philosophia o scientia. En la Introducción a la filosofía de Oliver Breton (Lafleur, I995, p. 478) se advierte que "filosofía, arte y ciencia poseen idéntica sustancia pero diferentes razones". Esta idea explica el por qué Blanquerna concluye su explicación acerca de la enseñanza de las artes y las ciencias diciendo que a partir del Arte se pueden alcanzar otras disciplinas como la filosofía o la medicina (Llull, 2009, p.26I). Por esto es necesario investigar los recursos textuales universitarios del siglo XII y XIII para conocer la semejanza entre artes, filosofía y ciencias. Esto es en gran parte la causa de las diversas obras en las que Ramon Llull ahondaba en una pluralidad disciplinas que representaban al Arte, gracias a la amplitud epistémica que aportan los principios y sus distintas aplicaciones.

La convergencia ars-philosophia se nota en la definición luliana de filosofía que posee elementos comunes con otras definiciones, ya que esta disciplina busca conocer las operaciones del creador (primam causam-creatoris), según las facultades humanas para tener acceso al conocimiento que se halla entre la iluminación (illuminatio) y la comprensión intelectual (assimilacio):

\begin{tabular}{|l|l|}
\hline $\begin{array}{l}\text { Philosophia est scientia, per quam intellectus } \\
\text { illuminatur ad cognoscendum primam causam }\end{array}$ & $\begin{array}{l}\text { Descripcio autem philosophiae ex proprietate } \\
\text { eius est quod philosophia est assimilacio ope- } \\
\text { primae effectum, et ad cognoscendum effectus } \\
\text { ribus creatoris gloriosi et sublimis secundum }\end{array}$ \\
$\begin{array}{l}\text { ralia et moralia. Liber de uniuersalibus (Llull, } \\
\text { I984, p. } 165 \text { ). }\end{array}$ & (Isaak ben Salomon Israeli, I938, p. 330). \\
\hline
\end{tabular}

Jean de Dacia cita la definición de Isaac Isareli y agrega: "opera enim creatoris perfecta sunt bona (...) per philosophicam autem disciplina perficitur homo et fit bonus". El sentido filosófico de ars es más evidente unas líneas antes cuando cita a Isidoro de Sevilla: "philosophia est ars artium, scientia secientiarum" (Jean de Dacia, I955, p. 6). El sentido universal de filosofía sustituye al ars y representa la fuente de los principios del conocimiento fruto de la especulación teológica, lo que es una fórmula repitida por los textos que circulaban en el studium medieval. Uno de los recursos que sirve de base a los textos citados es De divisione philosophiae de Gundisalvo en el que se repiten estas mismas definiciones (Baur, 7) a las que les acompañan las definiciones de las Artes liberales. Llull usa muchas de las expresiones de este texto para definir la gramática, que se explica como el arte que enseña a "hablar con corrección» (dretamente parlar-recte loquendi), o en la lógica, cuyo contenido son los conceptos universal-accidental (accidents/ 
universals-universalitas/accidentalitas). En el siguiente cuadro se observan las definiciones lulianas a la izquierda y las de Gundisalvo a la derecha:

\begin{tabular}{|c|c|c|}
\hline $\begin{array}{l}\text { Doctrina pueril, ed. J. San- } \\
\text { tanach (NEORLVII), Palma } \\
\text { de Mallorca: PRL, 2005. }\end{array}$ & $\begin{array}{l}\text { Arbor scientiae, ed. Pere } \\
\text { Villalba Varneda (ROL XXIV), } \\
\text { Turnhout: Brepols, } 2000 .\end{array}$ & $\begin{array}{l}\text { Dominicus Gundissalinus, } \\
\text { De divisione philosophiae, ed. } \\
\text { L. Baur, (BGPhM, } 4) \text {. }\end{array}$ \\
\hline $\begin{array}{l}\text { Gramatica es dreta[ment } \\
\text { parlar e] escriure }\end{array}$ & $\begin{array}{l}\text { Grammatica est ars, quae } \\
\text { docet ordinate loqui et accen- } \\
\text { tus ponere in uocabulis ut } \\
\text { oportet, et concordare uerba } \\
\text { et nomina. }\end{array}$ & $\begin{array}{l}\text { Grammatica est ars vel scien- } \\
\text { tia gnara recte loquendi, } \\
\text { recte scribendi. }\end{array}$ \\
\hline $\begin{array}{l}\text { Amable fil, per logica sabrás } \\
\text { conexer los jenres, les espe- } \\
\text { cies, les differencies, les } \\
\text { proprietats e los accidents, } \\
\text { qui son apelats los .v. univer- } \\
\text { sals; e per aquesta conaxensa } \\
\text { sabrás devalar de les cozes } \\
\text { generals a les especials. }\end{array}$ & $\begin{array}{l}\text { Et ad inuestigandum ista } \\
\text { principia, habet logicus inten- } \\
\text { tiones secundarias, quae sunt } \\
\text { rerum realium significata, de } \\
\text { quibus tractat naturalis, et ex } \\
\text { quibus logicus nomina trahit } \\
\text { et similitudines operationum } \\
\text { naturalium. }\end{array}$ & $\begin{array}{l}\text { Rebus autem, prout intelli- } \\
\text { guntur, accidit universalitas, } \\
\text { generalitas, specialitas, acci- } \\
\text { dentalitas et similia, propter } \\
\text { quae res, prout intelliguntur, } \\
\text { sunt genera, species et acci- } \\
\text { dentia et similia. }\end{array}$ \\
\hline $\begin{array}{l}\text { Retorica es parlar belament } \\
\text { e ordonada, per la qual son } \\
\text { agradablament oydes parau- } \\
\text { les e per la qual hom es exoit } \\
\text { moltes de vegades. }\end{array}$ & $\begin{array}{l}\text { Rhetoricus tractat, quod homo } \\
\text { loqui possit per uocabula } \\
\text { pulchra et ornata, ut sensus, } \\
\text { qui est auditus, placitum in } \\
\text { audiendo habere possit. }\end{array}$ & $\begin{array}{l}\text { Ars rhetorica est scientia } \\
\text { bene dicendi. Alii vero sic: } \\
\text { Rhetorica est scientia utendi } \\
\text { in privatis et publicis causis } \\
\text { plena et perfecta eloquentia. }\end{array}$ \\
\hline $\begin{array}{l}\text { Geomatria es doctrina de } \\
\text { formes immovables mon- } \\
\text { tiplicades en nombre en } \\
\text { humana pensa. }\end{array}$ & $\begin{array}{l}\text { Geometricus considerat quan- } \\
\text { titates continuas, et illas men- } \\
\text { surat de principio usque ad } \\
\text { medium, et de medio usque } \\
\text { ad finem, et facit circulum, } \\
\text { in quantum considerat in illo } \\
\text { medium, quod est centrum. }\end{array}$ & $\begin{array}{l}\text { Materia eius est magnitudo } \\
\text { immobilis, cuius alia est } \\
\text { linea, alia superficies, alia } \\
\text { corpus. Quae ideo mag- } \\
\text { nitudo immobilis dicitur, } \\
\text { quoniam abstracta a materia } \\
\text { omnino sine motu intelli- } \\
\text { guntur. }\end{array}$ \\
\hline $\begin{array}{l}\text { Arismetica es, fil, montiplicar } \\
\text { sumes en suma, e molts en } \\
\text { suma, e devesir .i. en moltz. }\end{array}$ & $\begin{array}{l}\text { Arithmeticus considerat dis- } \\
\text { cretas quantitates ex discretis } \\
\text { quantitatibus realibus mul- } \\
\text { tiplicatas in similitudinibus } \\
\text { phantasticis, ex quibus nume- } \\
\text { rum multiplicat, quem ponit } \\
\text { in figuris. }\end{array}$ & $\begin{array}{l}\text { Arithmetica est disciplina } \\
\text { quantitatis numerabilis } \\
\text { secundum se. Sive: Arithme- } \\
\text { tica est scientia de numero. }\end{array}$ \\
\hline
\end{tabular}




\begin{tabular}{|l|l|l|}
\hline $\begin{array}{l}\text { Musica es art per la qual } \\
\text { avem doctrina a cantar e } \\
\text { a sonar esturments dreta- } \\
\text { ment, e tost e espau, alsant e } \\
\text { baxant e agual los punts e les } \\
\text { veus en tal manera que sien } \\
\text { concordants diverses veus } \\
\text { e sons. }\end{array}$ & $\begin{array}{l}\text { Musicus considerat uoces } \\
\text { dispositas, scilicet quod sint } \\
\text { altae, infimae et mediocres, } \\
\text { longae et breues, grossae et } \\
\text { primae siue subtiles, propor- } \\
\text { tionatae ad accentus uocalium } \\
\text { et consonantium, ut uoces } \\
\text { ornare possit et melodiam } \\
\text { instrumentorum. }\end{array}$ & $\begin{array}{l}\text { Genus eius est, quod ipsa est } \\
\text { scientia harmoniacae modula- } \\
\text { tionis, quae ex concordantia } \\
\text { plurimorum sonorum vel } \\
\text { ex compositione vocum } \\
\text { conficitur. } \\
\text { Materia huius artis est } \\
\text { tonus. Tonus autem est } \\
\text { acuta enuntiatio vocis. Est } \\
\text { enim harmoniae differentia } \\
\text { et quantitas, quae in vocis } \\
\text { accentu et tenore consistit. }\end{array}$ \\
\hline $\begin{array}{l}\text { Estrolomia es demostrativa } \\
\text { sciencia per la qual ha hom } \\
\text { conaxensa que los corsos } \\
\text { celestials an seyoria e opera- } \\
\text { ció sobre los corsos terrenals. }\end{array}$ & $\begin{array}{l}\text { Astronomus considerat naturas } \\
\text { corporum supracaelestium, } \\
\text { influentias et impressiones, } \\
\text { quas mittunt in inferioribus } \\
\text { corporibus. }\end{array}$ & $\begin{array}{l}\text { Astronomia est scientia, quae } \\
\text { cursus et positionem stella- } \\
\text { rum secundum hominum } \\
\text { opinionem describit ad } \\
\text { temporum notitiam. }\end{array}$ \\
\hline
\end{tabular}

El estudio de las definiciones lulianas de las artes liberales podría elaborarse tal como en el cuadro anterior en el que se comparan obras con una cierta distancia temporal —Doctrina pueril (I276) y el Arbor sicientiae (I296) — junto a los textos que sirvieron de recurso a la enseñanza en el studium medieval —De divisione philosophiae. Es destacable el modo en que las disciplinas sermocinales mantienen una cierta continuidad en la obra luliana y tratan en general los mismos temas que menciona el texto de Gundisalvo. Sin embargo, Llull agrega el problema de las intenciones en la lógica, aunque este podría estar implicito en la disposición de las semejanzas (similitudo) entre conceptos, objetos y términos. En el apartado de geometría Llull se muestra más preocupado en el Arbor scientiae por la cuestión acerca de las construcciones geométricas con figuras — círculo, triángulo, cuadrado - para representar con ellas tanto el comportamiento elemental de la naturaleza como el discurrir del intelecto por los principios del Arte. En la aritmética sucede otro tanto, ya que Llull se interesa por la multiplicación y la suma de cantidades discretas. Es posible que dicho interes, que en apariencia no manifiesta Gundisalvo, pueda estar más cerca del tratado boeciano y que explique, en parte, el funcionamiento combinatorio de la tabula generalis. Respecto a la música sigue la distinción boeciana entre ejecución humana por medio de la voz y la ejecución instrumental, así como los parametros de tonalidad, armonía y consonancia. La mayoría de los detalles presentes en las definiciones lulianas de las artes liberales provienen de la tradición medieval, aunque matizadas por las cuestiones que más preocupaban al studium universitario durante el siglo XIII. 
Otro punto a destacar es que este mismo listado aparece en otras obras lulianas como el Ars generalis ultima o la Logica nova, lo que representa un tema recurrente en la evolución del pensamiento luliano. La cuestión a investigar es cómo las definiciones de las artes liberales, latentes en la tradición medieval modificaron la evolución del Arte en sus diversas transformaciones. Si observamos el primer ciclo de la obra luliana (Ars demonstrativa (I283) — Quatuor libri principiorum-) se nota claramente que el Arte respondía al modelo del studium medieval, así que ars es la uia que conduce a otras disciplinas, en especial la teología. Sin embargo, esta perspectiva cambia cuando el Arte se hace más robusto y puede agrupar los principios tanto de las artes liberales como de otras disciplinas como la medicina, el derecho y la filosofía natural. En el Ars inventiva y en versiones posteriores el Arte se convierte en la uia y la finalidad, ya que aparecen estrechamente unidas en el alfabeto luliano de principios. Esto explicaría que la lista de las definiciones de las artes y de las ciencias aparezcan en una misma obra junto a los principios del Arte luliano. La hipótesis de esta investigación es que es posible mostrar otro modo de justificar la evolución del Arte luliano, alejado de la excesiva formalidad combinatoria, por medio del significado y la definición de cada una de las artes liberales, lo que explicaría los grandes desafíos intelectuales e institucionales que Ramon Llull se propuso, por ejemplo la inserción del Arte en la enseñanza universitaria y su finalidad ético discursiva. Esta meta se apoyó en el impulso del dialogo sapiencial que se halla enraizado en las religiones del Mediterráneo y en la reflexión filosófica acerca de la pluralidad de las artes, la finalidad especulativa de la metafísica, la interpretación de la naturaleza, de la vida práctica y de la vida contemplativa.

La obra luliana que logra cristializar este objetivo de unidad entre artes y disciplinas reguladas por un mismo Arte es el Arbor scientiae. Dos siglos antes de ser escrita, circulaba entre los medievales un proverbio greco-latino que equiparaba scientia-ars con un árbol — siguiendo el símbolo del relato biblico — lo que fue recogido por Juan de Dacia como una evidente premonición del proyecto luliano del Ars major:

Ars est arbor, cuius radix est amarissima, fructus uero dulcissimus, et qui eius abhorret amaritudinem, eius nunquam gustabit dulcedinem (Jean de Dacia, 1955, p. 9).

BIBLIOGRAFÍA

Boecio, 2000: De sancta trinitate, en Opuscula theologica, ed. C. Moreschini, Munich.

Bonner A., 2007: The Art and Logic of Ramon Llull. A User's Guide, Leiden, Brill. 
Buenaventura, 1996: On the Reduction of the Arts to Theology (De reductione artium ad theologiam), ed. Z. Hayes, Nueva York.

Costurer, J., I700: Disertaciones históricas del culto inmemorial del B. Raymundo Lullio Dr. Iluminado y Mártir y de la inmunidad de censuras que goza su doctrina; con un apéndiz de su vida, Mallorca.

Diomedes, 1857 : Ars grammaticae libri III, ed. H. Keil (Grammatici latini I), Leipzig.

Dominicus Gundissalinus, 1903: De divisione philosophiae, ed. L. Baur, Munster.

Hillgarth J. N., I971: Ramon Lull and Lullism in fourteenth-century France, Oxford, Clarendon Press, p. 350, "Ars est collectio philosophum priorum et communis recta et rationalis deductio vel ordinatio ad finem inveniendam".

Isaak ben Salomon Israeli: 1938, Liber de definitionibus, ed. Muckle J. P., AHDLMA II.

Juan de Dacia, 1955, Divisio Scientiae, ed A. Otto, Copenhagen.

Lafleur, C., 1997: 'Les textes 'didascaliques' ('introductions à la philosophie' et 'guides de l'étudiant') de la faculté des arts de Paris au xime siècle: notabilia et status quaestionis", en L'enseignement des disciplines à la Faculté des arts (Paris et Oxford, XIII-XV siècles), ed. O. Weijers y L. Holtz, Turnhout, p. 345-372.

Le Goff, J., 1957: Les intellectuels au Moyen Age, París,

Leff, G., I992-I994: "The trivium and the Three Philosophies"; North, J., "The quadrivium", en A History of the University in Europe I: Universities in the Middle Ages, ed. Ridder-Symoens, Cambridge, pp. 306-358.

Llull, R., 2009: Romanç d'Evast e Blanquerna, ed. A. Soler y J. Santanach, Palma.

Oliver Breton, 1997: "Philosophia magistri Oliueri Britonis", en L'enseignement de la philosophie au XIIIe siecle : autour du Guide de l'etudiant du ms. Ripoll rog, ed. J. Carrier y C. Lafleur, Turnhout, pp. 474-487.

Ramon Llull, 1984: Liber de uniuersalibus, ed. A. Madre (ROL XII).

—, 2009: Doctrina puerili, ed. J. Medina (ROL XXXIII), Turnhout. 
